\title{
Scalable Knowledge Extraction and Visualization for Web Intelligence
}

\author{
Arno Scharl \\ webLyzard technology \& \\ MODUL University Vienna \\ scharl@,weblyzard.com
}

\author{
Albert Weichselbraun \\ webLyzard technology \& \\ University of Applied Sciences Chur \\ weichselbraun@weblyzard.com
}

\author{
Max Göbel \\ Vienna University of Economics \\ and Business \\ max.goebel@wu.ac.at
}

\author{
Walter Rafelsberger \\ webLyzard technology \\ rafelsberger@weblyzard.com
}

\author{
Ruslan Kamolov \\ MODUL University Vienna \\ ruslan.kamolov@modul.ac.at
}

\begin{abstract}
Understanding stakeholder perceptions and assessing the impact of campaigns are key questions of communication experts. Web intelligence platforms help to answer such questions, provided that they are scalable enough to analyze and visualize information flows from volatile online sources in real time. This paper presents a distributed architecture for aggregating Web content repositories from Web sites and social media streams, memory-efficient methods to extract factual and affective knowledge, and interactive visualization techniques to explore the extracted knowledge. The presented examples stem from the Media Watch on Climate Change, a public Web portal that aggregates environmental content from a range of online sources.
\end{abstract}

\section{Introduction}

Media analytics applications have been developed for various domains including sports [7], politics [2] and climate change [10], often focusing on specific aspects such as event detection [1] or the annotation of video broadcasts [2]. To provide actionable knowledge and support decisions, such media analytics systems face two major challenges:

- compile and annotate large document collections from online sources - heterogeneous in terms of authorship, formatting, style (e.g. news article vs. tweets), and update frequency;

- provide a visual user interface to select the most relevant subsets of the information space, and to analyze and visualize the extracted information.

Contextual information, when properly disambiguated, plays a vital part in addressing both challenges and can improve several steps in the processing pipelines of media analytics platforms. Contextual information can guide content acquisition via focused crawling [6], increase the accuracy of knowledge ex- traction algorithms, and help to understand the role of affective knowledge in human decision-making [4].

The Media Watch on Climate Change (MWCC) ${ }^{1}$ and the U.S. Climate Resilience Toolkit (CRT) ${ }^{2}$ are two public showcases of the technologies presented in this article. Shown in Figure 1, MWCC is a content aggregator on climate change and related environmental issues [10], while CRT was developed in response to U.S. President Barack Obama's Climate Action Plan to provide expert knowledge and a suite of analytic tools to help citizens and communities manage climaterelated risks and opportunities [3].

Using the distributed processing architecture of the webLyzard Web intelligence and media analytics platform [26] shown in Figure 2, both applications compile large archives of relevant Web content from multiple online sources, and provide visual tools to access and analyze this content [10].

MWCC has provided the data feeds for the examples presented in this paper. The portal aggregates multilingual content from English, French and German online sources - social media including Facebook, Twitter, Google+ and YouTube, and the Web sites of news agencies, Fortune 1000 companies and environmental NGOs. Exploring the role of user-generated content archives as a "knowledge commons" [9], MWCC is currently being extended into a collective awareness platform through the DecarboNet.eu project, with a focus on knowledge co-creation processes.

The remainder of this paper is structured as follows: Section 2 describes the system architecture including queue management, virtualization and content filtering techniques. Section 3 presents knowledge extraction techniques and algorithmic optimizations that enable to process the gathered Web content in real time, and to create a contextualized information space spanning geospatial, temporal and social dimensions.

\footnotetext{
${ }^{1} \mathrm{http}: / /$ www.ecoresearch.net/climate

2 http://toolkit.climate.gov
} 


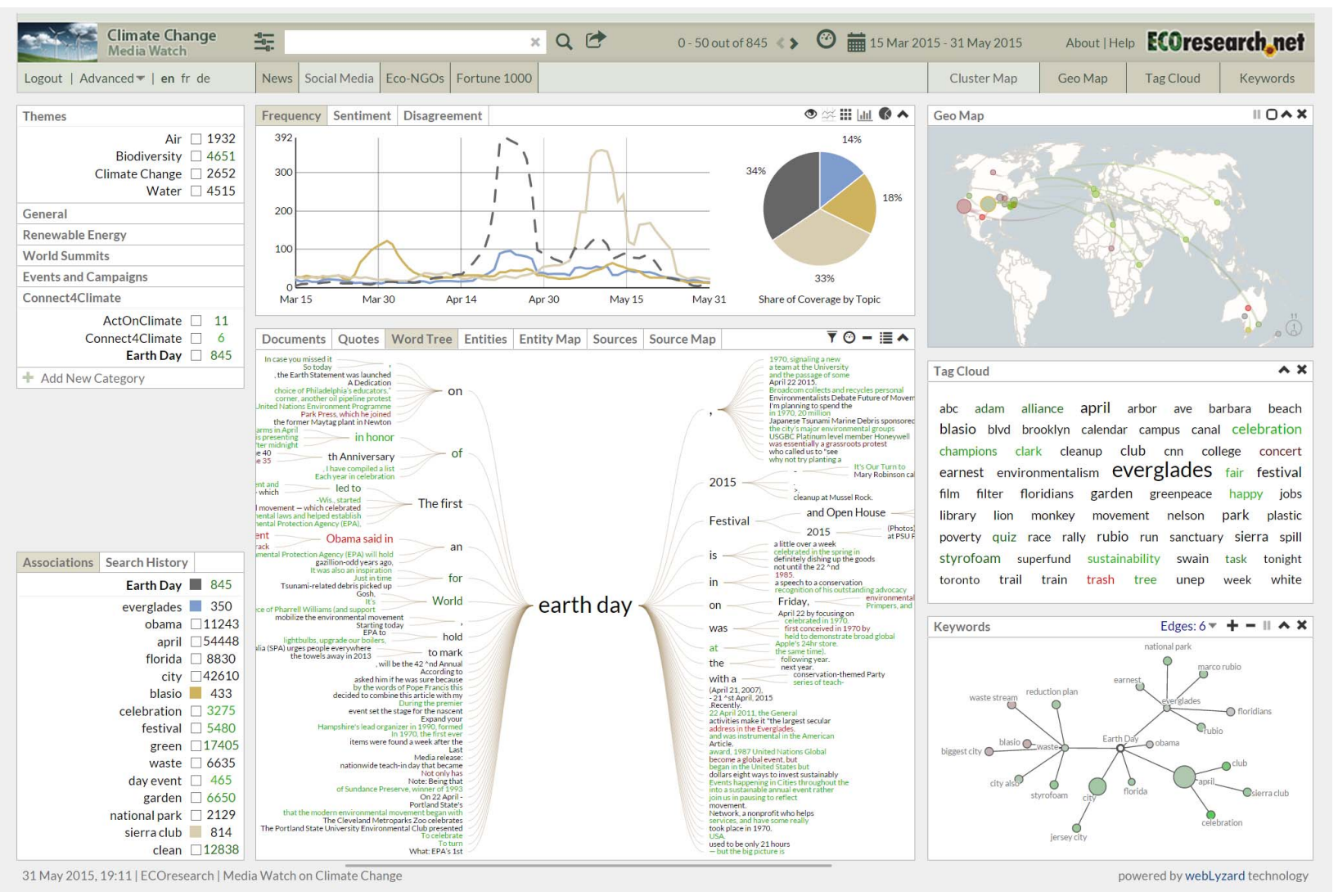

Figure 1. Visual dashboard of the Media Watch on Climate Change (www.ecoresearch.net/climate)

Methods to analyze and visualize this information space presented in Section 4 shed light on stakeholder perceptions, reveal information flows, and provide indicators for assessing the impact of environmental education and awareness campaigns.

\section{Content Acquisition and Preprocessing}

Figure 2 illustrates the content acquisition pipeline. The system pursues a hybrid strategy of real-time updates from streaming APIs and focused crawling of Web content by processing and enriching data from unstructured and structured evidence sources.

A dynamic task scheduling component uses the MWCC portal configuration to allocate resources to content acquisition and preprocessing tasks - i.e., focused crawls of Web sites and RSS feeds, real-time updates of social media sites, or keyword analyses.

The webLyzard platform uses the scalable and flexible messaging broker service RabbitMQ [25] to distribute these tasks to worker instances, which perform the content acquisition and processing tasks. Kernelbased Virtual Machines [22] and Docker [18] images enable the dynamic creation and destruction of worker instances based on the current system load and number of pending tasks. Although virtualization ensures a scalable content acquisition and processing pipeline, additional steps are taken to manage the volume and dynamic nature of online content. Efficient preprocessing and filtering removes irrelevant content at an early stage of the processing pipeline. This reduces the number of documents that later need to be processed by the computationally more expensive knowledge extraction algorithms (see Section 3).

Boilerplate detection, if required, identifies overview pages and extracts the main content from news articles, blogs and Web pages. Locality sensitive hashing [8] to identify near duplicates and a domain specificity measure allow removing irrelevant content at an early stage. The domain specificity measure uses a combination of blacklists and whitelists to assess the relevance of gathered documents in the context of the targeted domain - i.e., climate change and related environmental issues in the case of the MWCC portal.

\section{Knowledge Extraction}

Which environmental organizations attract significant media attention and have a positive reputation among social media users? Who are the most respected climate scientists and what are mainstream news media associating with their recent public appearances? 


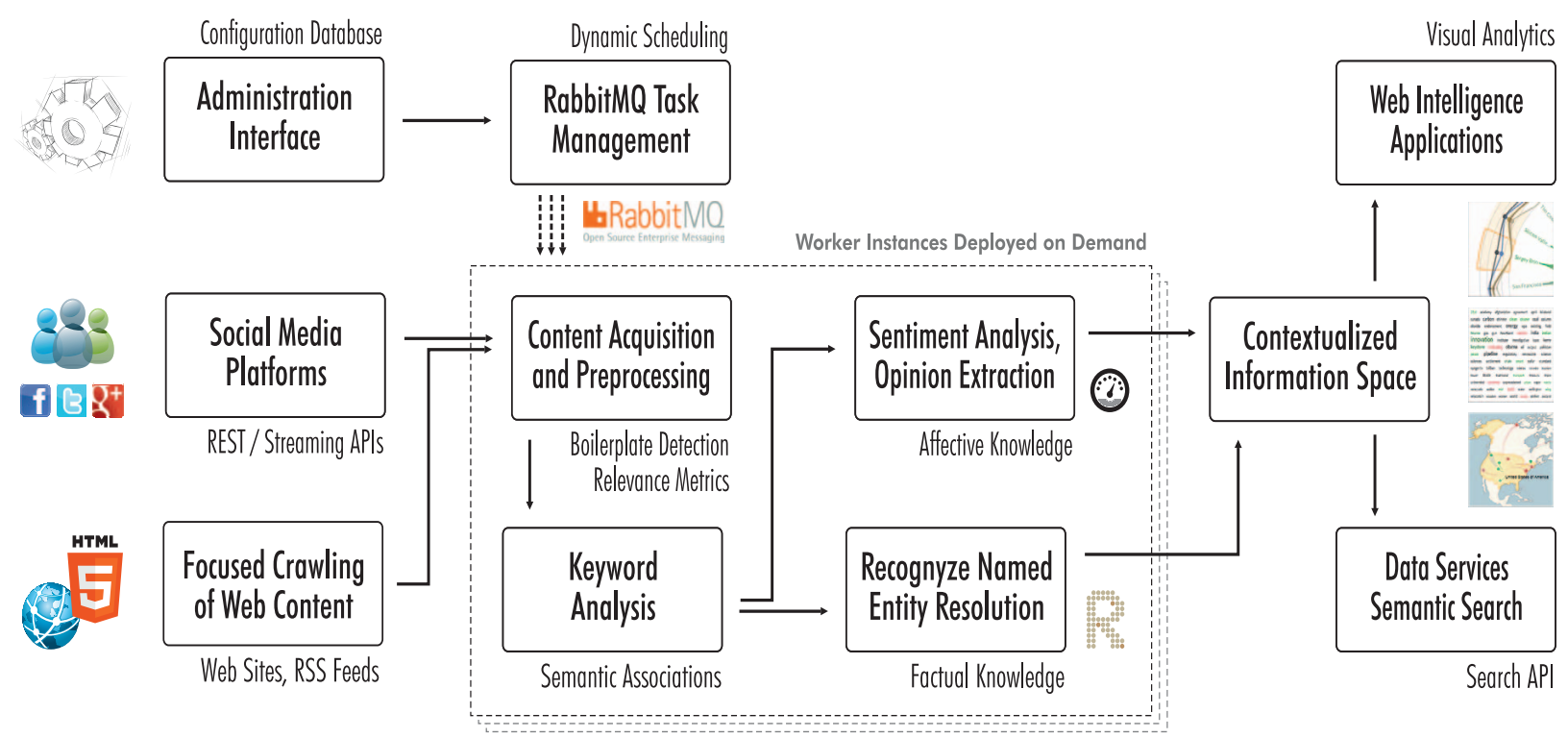

Figure 2. Overview of the distributed processing architecture of the weblyzard platform

For properly answering such questions, the Media Watch on Climate Change needs to extract both factual as well as affective knowledge.

\subsection{Factual versus Affective Knowledge}

Factual Knowledge includes concepts, instances, and relations among identified entities. MWCC utilizes Recognyze [13], a named entity recognition and resolution component that draws upon structured external knowledge repositories such as DBpedia [16], Freebase [19] and GeoNames [20] to identify, classify and disambiguate named entities - people, organizations, and locations. Confidence values align entities with external knowledge repositories or corporate databases, resulting in a continuously evolving knowledge repository that helps to better understand networks and the dynamic relations [1] among their actors.

Affective Knowledge includes sentiment and other emotions expressed in a document, which are captured and evaluated by opinion mining algorithms [11;12]. Lexical methods rely on sentiment lexicons, which contain known sentiment terms and their respective sentiment values. The ratio of positive and negative terms in a document is a common indicator of overall polarity that is often used for classifiers. Even when considering negations and intensifiers, such methods are computationally inexpensive.

More advanced algorithms rely on dependency parsing or integrate external semantic knowledge bases. This significantly increases the computational demands and calls for more effective approaches to store, retrieve and analyze Web content (see Section 3.2).
Combining factual and affective knowledge creates synergies - the factual knowledge of Recognyze, for example, helps to contextualize the sentiment analysis process, to process ambiguous sentiment terms, and to detect opinion holders and opinion targets.

\subsection{Ensuring Maximum Scalability}

The extraction of knowledge from unstructured Web content repositories benefits from distributed processing strategies that allocate computational resources dynamically, taking into account the nature of a given task and the current status of the infrastructure. Further performance gains can be achieved by optimizing the storage, integration and analysis of lexicons and external databases, and by using the derived artifacts for real-time text searches. A particularly well-suited data structure for performing these operations are Directed Acyclic Word Graphs (DAWGs), which in contrast to prefix trees [5] efficiently encode suffixes as well as prefixes and support fast and efficient searches.

Figure 3 shows the DAWG representation of the following ten words: success, successes, successful, successfully, successfulness, succession, successions, successive, successively and successiveness. In this example, the common prefix "success" is represented in a single node. Colored nodes indicate word ends and following the node structure to a colored node yields the strings, encoded in the corresponding path.

The DAWG representation allows efficient string searches, covering all encoded terms in parallel. A search compares a string sequence $\mathrm{s}=\left\{\mathrm{s}_{1}, \ldots \mathrm{s}_{\mathrm{m}}\right\}$ with the letters available in the graph structure $g=\left\{n_{1 i}\right.$, $\left.\ldots \mathrm{n}_{\mathrm{mi}}\right\}$, requiring one comparison per letter. 


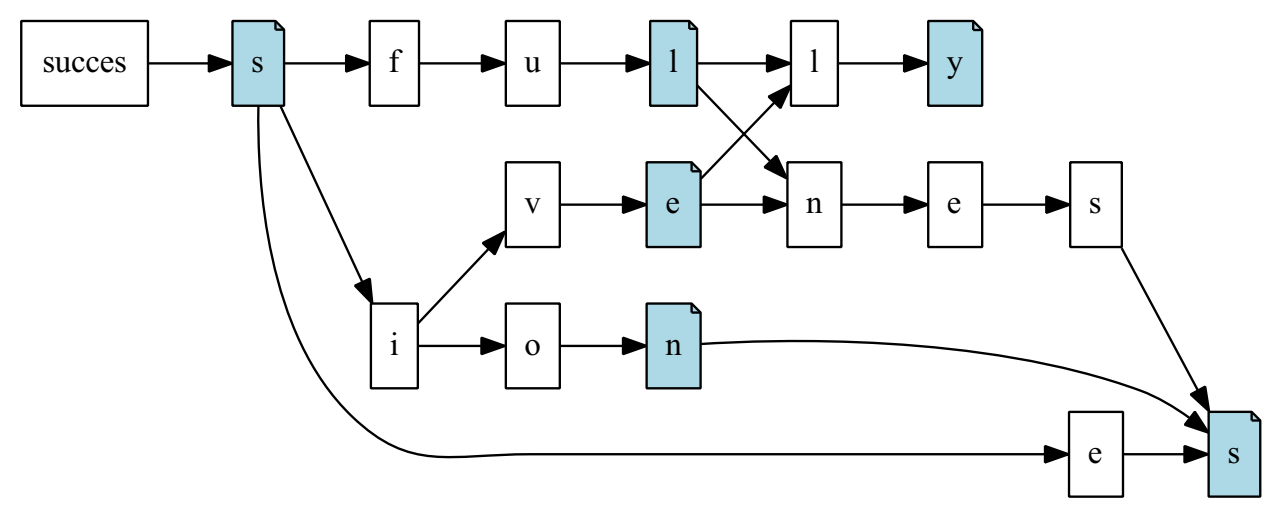

Figure 3. Visual representation of a Directed Acyclic Word Graph (DAWG)

If the graph contains a node for every letter of the string sequence, and the final node $\mathrm{n}_{\mathrm{mi}}$ encodes a word end, the search has been successful. The search time is only influenced by the length of the strings encoded in the DAWG, but not by the number of strings.

The Recognyze component also cascades DAWG representations for disambiguation - e.g., by combin-

ing searches for ambiguous company names with words which typically indicate a company name such as "Inc.", or "Corp.". Recognyze provides the ".jet" format for building and serializing this data structure.

Table 1 compares the performance and storage requirements of the .jet representation with the standard Java implementations ArrayList, HashSet and TreeSet. The experiments draw upon the English, French and German versions of the hunspell lexicon [21], which has been extended using the unmunch utility included in the hunspell distribution. In addition, a German lexicon (de_chr) obtained from the Chromium project [15] has been used in the experiments. These lexicons are also used in Recognyze for pre-processing and classifying company names. All tests have been performed on an HP ProLiant Server with 128 GB RAM, a 32-core Intel Xeon E5-2650 2.00 GHz CPU and OpenJDK $7 \mathrm{u} 65$ (using a single core to increase reliability).

Table 1 reflects the memory efficiency of the jet format, especially when used to encode large dictionaries such as the German Chromium lexicon (de_chr). In such cases, the .jet format even outperforms compression algorithms such as gzip and bzip2. These results are particularly noteworthy when considering lookup performance. The listed lookup times have been determined based on a randomly chosen, balanced sample of 500,000 set items and 500,000 items that are not part of the set. As expected, the lookup times for lists cannot compete with speeds obtained from sets and the .jet format. The .jet format's performance lies between the HashSet and a TreeSet, yet it provides a much leaner representation of the lookup strings in terms of memory consumption. Although the HashSet performs better for single lookups, it does not support parallel searches for all strings encoded in the HashSet which is a key feature of the DAWG representation.

Table 1. Comparative evaluation of the .jet format using a Directed Acyclic Word Graph (DAWG) representation

\begin{tabular}{|c|c|c|c|c|c|}
\hline \multicolumn{6}{|c|}{ On Disk Size (in MB) } \\
\hline Lexicon & Entries & Size &. $\mathrm{gz}$ & .bz2 & .jet \\
\hline de & 630,516 & 8.8 & 1.7 & 2.0 & 1.4 \\
\hline de chr & $12,238,334$ & 195.0 & 30.0 & 30.0 & 6.5 \\
\hline en & 112,931 & 1.2 & 0.3 & 0.3 & 0.6 \\
\hline $\mathrm{fr}$ & 382,441 & 4.7 & 0.9 & 1.1 & 0.7 \\
\hline \multicolumn{6}{|c|}{ Memory Size (in MB) } \\
\hline Lexicon & Entries & List & HashSet & TreeSet & .jet \\
\hline de & 630,516 & 45 & 67 & 67 & 1 \\
\hline de_chr & $12,238,334$ & 951 & 1361 & 1391 & 6 \\
\hline en & 112,931 & 7 & 11 & 11 & $<1$ \\
\hline $\mathrm{fr}$ & 382,441 & 25 & 38 & 39 & $<1$ \\
\hline
\end{tabular}




\begin{tabular}{|l|r|r|r|r|r|}
\hline \multicolumn{2}{|l|}{ Lookup Time for 1 Million Entries (in ms) } \\
\hline Lexicon & Entries & List & HashSet & TreeSet & .jet \\
\hline de & 630,516 & $>1 \mathrm{E} 6$ & 209 & 1237 & 626 \\
\hline de_chr & $12,238,334$ & $>1 \mathrm{E} 6$ & 251 & 2428 & 694 \\
\hline en & 112,931 & $>1 \mathrm{E} 6$ & 55 & 569 & 452 \\
\hline $\mathrm{fr}$ & 382,441 & $>1 \mathrm{E} 6$ & 88 & 912 & 404 \\
\hline
\end{tabular}

\section{Visualizing the Extracted Knowledge}

The scalability gains described in the preceding section allow the real-time extraction of knowledge from Web-scale content streams. Operating on such content streams, the MWCC visual dashboard [10] shown in Figure 1 reveals popular issues being discussed in conjunction with specific topics. The various dashboard components combine factual and affective knowledge to reveal the context of online discussions. This includes interactive trend charts for the temporal context of frequency and sentiment data (Section 4.1), a geographic map for regional context (Section 4.2), and an entity map to depict relations among identified people, organizations and locations (Section 4.3).

Across all these visualizations, color coding conveys affective knowledge - i.e., average document sentiment ranging from green (positive) to grey (neutral) and red (negative).

The presented examples are based on more than 125,000 documents about Earth Day 2015 - from 150 Anglo-American news media sites, blogs, Web 2.0 platforms (including Twitter, Facebook, Google+, and YouTube), scientific outlets, and the websites of environmental organizations and Fortune 1000 companies.

\subsection{Trend Chart}

The trend chart shown in Figure 3 reflects the evolution of topics. Whenever a different combination of sources or topics is selected, dynamic transitions show how these changes affect the displayed data set. Hovering above data points displays tooltips with weekly content statistics and associated keywords. This helps to investigate an issue and identify who or what has triggered an observable trend. A sentiment filter allows restricting the analysis to either positive or negative online coverage. The entity map presented in Section 4.3 uses a circular version of the trend chart to depict semantic associations between topics and entities.

\subsection{Geographic Map}

Once a user has entered a search query, MWCC ranks the matching results by relevance, date, or geographic location. The results are projected onto a geographic map that shows the regional distribution of Web content - e.g., references to locations that cooccur with the term "earth day" as shown in Figure 4. The position of circles is determined by the geographic coordinates of these references, their size is proportional to the number of matches for a specific location.
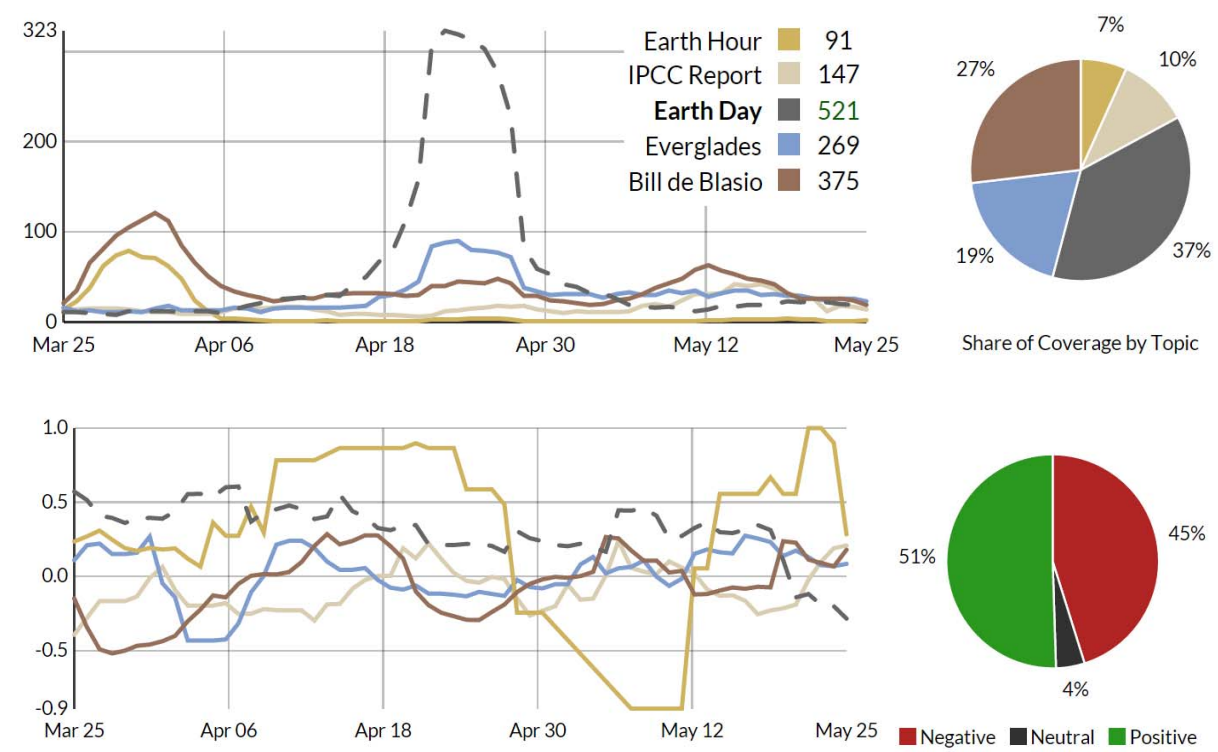

Figure 4. Trend charts showing frequency (above) and sentiment (below) of Earth Day news media coverage between March and May 2015 


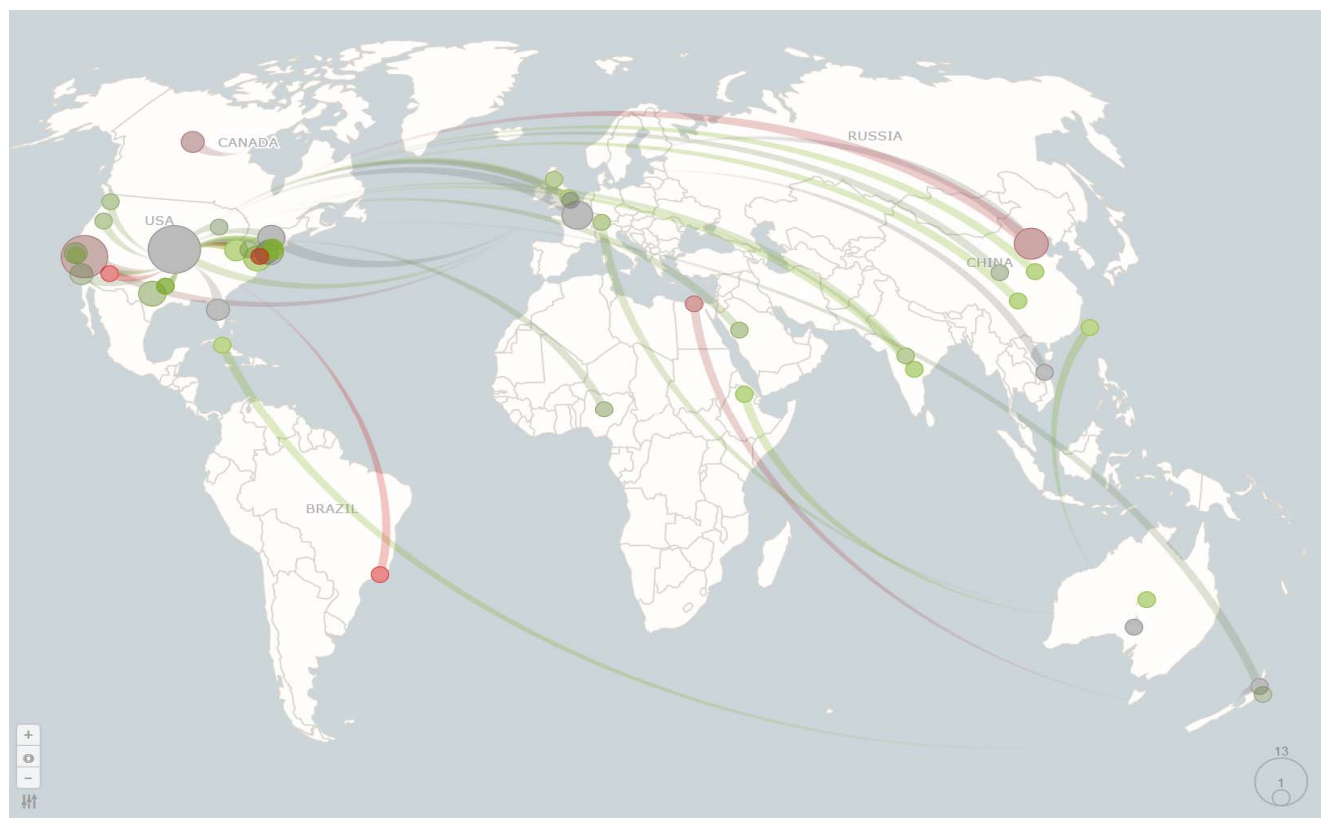

\begin{tabular}{|c|c|c|c|c|c|c|c|}
\hline Documents & Quotes & Word Tree & Entities & Entity Map & Sources & Source Map & 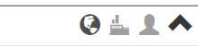 \\
\hline \multicolumn{4}{|l|}{ Location } & Count $\nabla$ & Latitu & ude Longitude & Sentiment \\
\hline \multicolumn{4}{|c|}{$\begin{array}{l}\text { United States } \\
\text { earth day | everglades | obama }\end{array}$} & 427 & 39.8 & -98.5 & +0.2 \\
\hline \multicolumn{4}{|c|}{$\begin{array}{l}\text { Florida } \\
\text { everglades | earth day | obama }\end{array}$} & 97 & 28. & -82.5 & -0.0 \\
\hline \multicolumn{4}{|c|}{$\begin{array}{l}\text { New York } \\
\text { earth day | blasio | waste }\end{array}$} & 96 & 43. & -75.5 & +0.1 \\
\hline \multicolumn{4}{|c|}{$\begin{array}{l}\text { Washington, D. C. } \\
\text { earth day | everglades | obama }\end{array}$} & 87 & 38. & -77.0 & +0.1 \\
\hline \multicolumn{4}{|c|}{$\begin{array}{l}\text { California } \\
\text { earth day | santa barbara | barbara }\end{array}$} & 62 & 37. & -119.8 & +0.2 \\
\hline \multicolumn{4}{|c|}{$\begin{array}{l}\text { United Kingdom } \\
\text { earth day | april | garden }\end{array}$} & 61 & 54.8 & -2.7 & +0.3 \\
\hline
\end{tabular}

Figure 5. Geographic map and references to locations in conjunction with the search term "earth day" in Anglo-American news media coverage between March and May 2015

When rendering documents in their geospatial context, the system distinguishes between source and target information - i.e., the authors' locations versus the primary locations referenced in the documents, which is determined by applying the above mentioned Recognyze component to a geo-tagging process (the table underneath the map shows a list of the identified geographic entities, sorted by decreasing co-occurrence frequency with "earth day"; the grey keywords underneath each location name are semantic associations determined through co-occurrence analysis).

\subsection{Entity Map}

To identify opinion leaders and reveal key factors influencing social conversations about a topic, the webLyzard platform detects not only locations, but also other named entities such as people and organizations that have an impact on news and social media coverage. To develop a deeper understanding of this process, analysts must not only understand how these entities influence topics of interest, but also unravel the interconnected relations among the entities themselves.

How did a public appearance of climate scientist impact the perception of an issue, for example, or what are journalists associating with the government's latest policy announcement? To help answer such questions, the Entity Map shown in Figure 5 visualizes (i) relations among named entities in the analyzed corpus, and (ii) co-occurrence patterns between these entities and user-defined search terms. Based on news media coverage between March and May 2015, the list of referenced entities includes politicians such as U.S. President Barack Obama and former Vice President $A l$ Gore, and organizations such as Twitter and $H \& M$. The Entity Map component combines a radial convergence diagram and a line chart with radial imposition. 


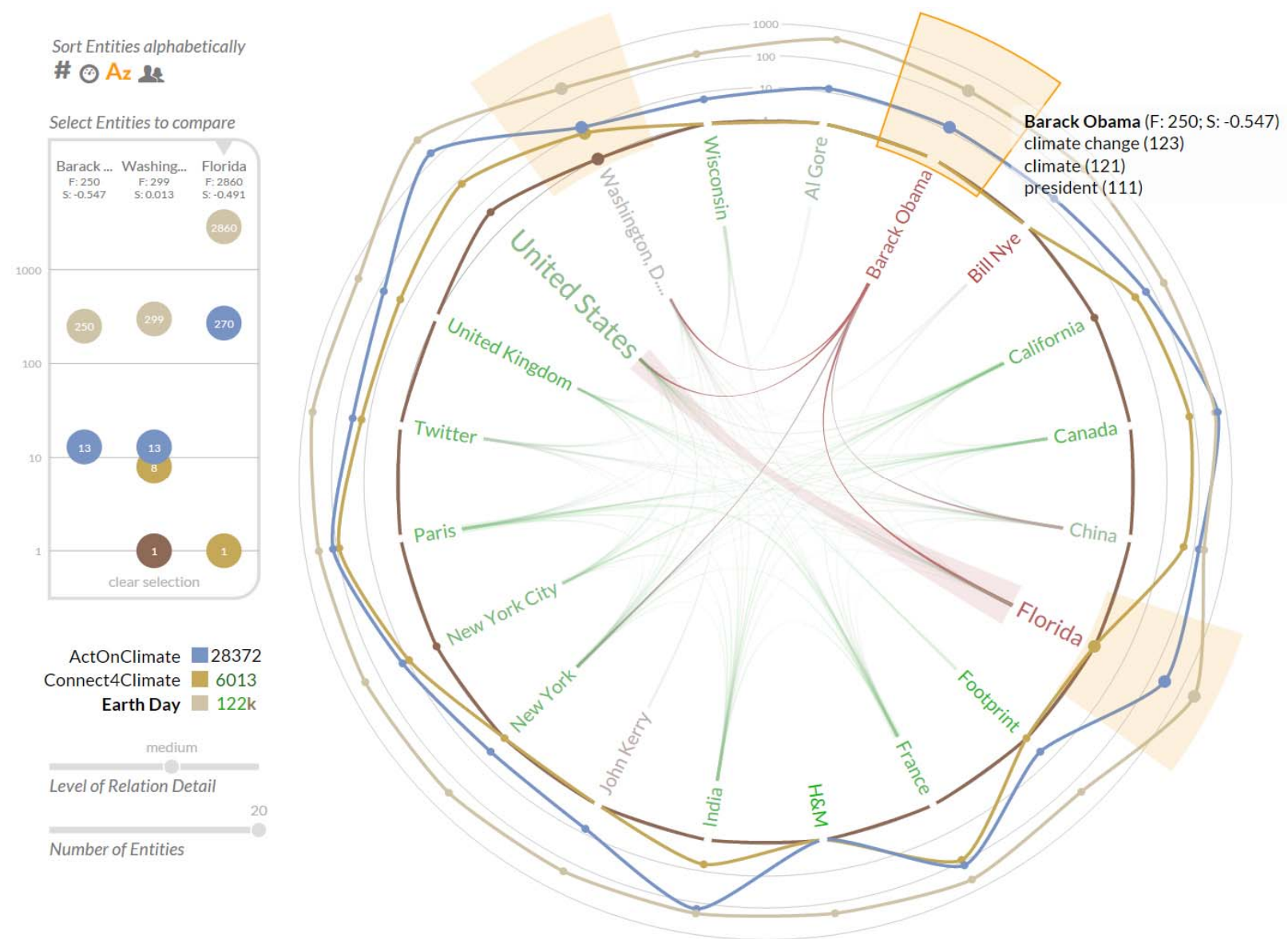

Figure 6. Entity Map showing people and organizations related to the search term "earth day" in AngloAmerican news media coverage between March and May 2015, the relation strength among those entities, and their association with selected topics.

Radial Convergence Diagram. Located in the center of the graph, the radial convergence diagram displays relations among different entities using ribbons. Entity names are displayed along a circle - their font size indicates the number of documents that mention the entity, their color ranges from red to green depending on the average sentiment (in line with the sentiment color coding of the word tree and the geographic map).

The thickness of an arc represents the number of co-occurrences between an entity pair. On mouse-over, the opacity of arcs that connect the selected entity to other entities is increased. A slider element in the lower left corner controls the level of detail in the radial convergence diagram - i.e., determines the threshold for showing relations among entities. The second slider in the lower left corner adjusts the number of entities to be shown, between a minimum of five and a maximum of 20 entities.

Line Chart. Surrounding the radial convergence diagram in the center, the data points in the line chart show the number of co-occurrences between an entity and the selected topics - e.g., Earth Day, ActOnClimate and Connect4Climate in Figure 5. To increase readability and facilitate comparisons across topics, the line chart uses a logarithmic scale.

Both components are being updated via animated transitions. Users can select which entity types to display (people, organizations and locations) and sort them by (i) the number of documents which reference the entity in descending order, (ii) the average sentiment of documents containing the entity, from positive to negative, (iii) alphabetically by name, and (iv) by entity type. Hovering over entities highlights the corresponding sector, shows a tooltip with semantic associations, and highlights the arcs in the radial convergence diagram. Clicking on an entity causes supplemental information to be displayed in a sidebar, which uses logarithmic scale to show data points with cooccurrence values and additional information about the selected entity. The sidebar allows comparing the three most recently selected entities, which remain highlighted in the graph. 


\section{Summary and Conclusion}

The Web intelligence platform presented in this paper is a big data application for the real-time analysis of Web-scale content streams - extracting factual and affective knowledge from these collections, and establishing relations between identified topics and named entities such as people, organizations, and locations. The Media Watch on Climate Change [23] is a public showcase of this platform that aggregates multilingual Web content from English, French and German Web sites (news media, Fortune 1000 companies, municipalities and environmental organizations), and from social media platforms including Twitter, Facebook, Google+ and YouTube.

The Recognyze named entity resolution component analyzes the collected text resources to identify named entities. Disambiguation algorithms then align these entities with related items in external structured repositories such as DBpedia.org, Freebase.com and GeoNames.org. To achieve the required scalability for encoding and processing the massive amounts of data contained in such repositories, a distributed processing strategy couples virtualization and dynamic resource allocation with optimized data structures based on $d i$ rected acyclic word graphs for managing lexicons and external databases.

Interactive visualizations allow users to explore the resulting information space, combining factual and affective knowledge to reveal contextual information in online discussions. The presented examples include trend charts to depict the temporal context of Web coverage, a geospatial projection to show its regional distribution, and an entity map to explore relations between referenced topics and entities.

The extraction and visualization of context information transforms collections of Web content into valuable repositories of actionable knowledge. This knowledge helps analyst to better understand the impact of communication campaigns on public opinion and decision making on both individual and collective levels. Thereby, the presented techniques benefit a wide range of organizations including enterprises, nongovernment entities, news media outlets, science agencies, and policy makers. Revealing online trends can help these organizations to adopt better strategies for engaging audiences, guide their communication and public outreach campaigns, and increase the effectiveness of their decision making processes.

Three European research initiatives are currently extending the Web intelligence and visual analytics platform presented in this paper. The ASAP Project [14] increases the scalability of the underlying methods, while the PHEME Project [24] enriches their functionality by adding veracity detection to reveal online myths and rumors. The DecarboNet Project [17] extends the Media Watch on Climate Change into a collective awareness platform that supports collaborative editing with real-time content recommendations, and reveals how information is created and shared in communities of environmental stakeholders.

Acknowledgement. The work presented in this paper has been conducted as part of the research projects DecarboNet.eu, PHEME.eu and ASAP-FP7.eu, which have received funding by the European Union's 7th Framework Program for research, technology development and demonstration under the Grant Agreements No. 610829, 611233 and 619706; and the Radar Media Criticism Switzerland project funded by the Swiss National Science Foundation (No. 150327).

\section{References}

1. Adams, B., Phung, D. and Venkatesh, S. (2011). Eventscapes: Visualizing Events Over Times with Emotive Facets. 19th ACM International Conference on Multimedia (MM-2011). Scottsdale, USA: 1477-1480.

2. Diakopoulos, N., Naaman, M. and Kivran-Swaine, F. (2010). Diamonds in the Rough: Social Media Visual Analytics for Journalistic Inquiry. IEEE Symposium on Visual Analytics Science and Technology (VAST-2010). Salt Lake City, USA: IEEE: 115-122

3. Duffy, P.B., Colohan, P., et al. (2014). "Empowering America's Communities to Prepare for the Effects of Climate Change: Developing Actionable Climate Science Under the President's Climate Action Plan", American Geophysical Union, Fall Meeting 2014. San Francisco, USA. A1.

4. Hoang, T.-A., Cohen, W.W., et al. (2013). Politics, Sharing and Emotion in Microblogs. IEEE/ACM International Conference on Advances in Social Networks Analysis and Mining. Niagara Falls, Canada: ACM Press: 282-289.

5. Knuth, D.E. (1997). The Art of Computer Programming, Volume 3: Sorting and Searching (2nd ed). Upper Saddle River: Addison-Wesley.

6. Mangaravite, V., Assis, G.T.d. and Ferreira, A.A. (2012). Improving the Efficiency of a Genre-aware Approach to Focused Crawling Based on Link Context. Eighth Latin American Web Congress (LA-WEB 2012). Cartagena de Indias, Colombia: IEEE CPS: 17-23.

7. Marcus, A., Bernstein, M.S., et al. (2011). Twitinfo: Aggregating and Visualizing Microblogs for Event Exploration. 2011 Annual Conference on Human Factors in Computing Systems (CHI-11). Vancouver, Canada: ACM: 227-236.

8. Paulevé, L., Jégou, H. and Amsaleg, L. (2010). "Locality Sensitive Hashing: A Comparison of Hash Function Types and Querying Mechanisms", Pattern Recognition Letters, 31(11): 1348-1358. 
9. Pitt, J., Bourazeri, A., et al. (2013). "Transforming Big Data into Collective Awareness", IEEE Computer, 46(6): 40-45.

10. Scharl, A., Hubmann-Haidvogel, A., et al. (2013). "From Web Intelligence to Knowledge Co-Creation - A Platform to Analyze and Support Stakeholder Communication", IEEE Internet Computing, 17(5): 21-29.

11. Weichselbraun, A., Gindl, S. and Scharl, A. (2013). "Extracting and Grounding Contextualized Sentiment Lexicons”, IEEE Intelligent Systems, 28(2): 39-46.

12. Weichselbraun, A., Gindl, S. and Scharl, A. (2014). "Enriching Semantic Knowledge Bases for Opinion Mining in Big Data Applications", Knowledge-Based Systems, 69: 78-86.

13. Weichselbraun, A., Streiff, D. and Scharl, A. (2014). Linked Enterprise Data for Fine Grained Named Entity Linking and Web Intelligence. 4th International Conference on Web Intelligence, Mining and Semantics (WIMS-2014). Thessaloniki, Greece: ACM Press.
Online Resources

14. ASAP Project $\mid$ www.asap-fp7.eu

15. Chromium Project $\mid$ www.chromium.org

16. DBpedia $\mid$ www.dbpedia.org

17. DecarboNet $\mid$ www.decarbonet.eu

18. Docker | www.docker.io

19. Freebase / www. freebase.com

20. GeoNames | www.geonames.org

21. Hunspell Spell Checker | hunspell.sourceforge.net

22. Kernel Virtual Machine | www.linux-kvm.org

23. Media Watch on Climate Change | www.ecoresearch.net/climate

24. PHEME Project | www.pheme.eu

25. RabbitMQ | www.rabbitmq.com

26. webLyzard |www.webLyzard.com 